\title{
Dynamic characteristics modelling and adaptability research of the balise transmission module in high speed railways
}

\author{
H. Zhao ${ }^{1}, \mathrm{~S} . \operatorname{Sun}^{1} \& \mathrm{~W} . \mathrm{Li}^{2}$ \\ ${ }^{1}$ School of Electronic Information and Engineering, \\ Beijing Jiaotong University, China \\ ${ }^{2}$ Beijing Jiaoda Signal Technology Co., Ltd, China
}

\begin{abstract}
General simulation requirements within the train control system simulation framework were described. Dynamic characteristics were modelled at four resolution layers, ranging from low to very high resolution. The research focuses mainly on the dynamic behaviour and simulation representation at the signal emulation layer. The Balise Transmission Module Hardware-In-the-Loop simulator was justified with respect to dynamic behaviour modelling. Furthermore, the relationship between Balise Transmission Module dynamic characteristics and train speed was verified by simulation and test data. The speed factor is derived and analyzed. Finally, quantitative evaluation issues of high-speed adaptability were explored based on the deduction of decoding failure probability under a certain bit error rate and the availability targets for a certain line.
\end{abstract}

Keywords: Balise Transmission Module, dynamic characteristics, adaptability, high speed railway.

\section{Introduction}

Balise Transmission Module (BTM) is a part of the train control system onboard constituent, and has the main functions of generating tele-powering signals to the balise, to receive and process up-link signals from the balise. BTM has been successfully used in China Passenger Dedicated Lines (DPL) with the maximum speed of $250 \mathrm{Km} / \mathrm{h}$ and high-speed lines with a maximum speed of 
$350 \mathrm{Km} / \mathrm{h}$, such as the Beijing-Tianjin DPL and Wuhan-Guangzhou DPL KeJiYun[2008] NO.143 [1]. Valuable test data and operation experience were acquired with respect to the BTM used under the high-speed operation conditions. However, Eurobalise specification recommended that the maximum applicable speed for the reduced size balise is only $300 \mathrm{Km} / \mathrm{h}$, as recommended in SUBSET-036-V2.4.1 [2]. Issues about BTM static feature simulation, test and application rules were studied in the past (Yang et al. [3], Zhen and Zhao [4] and Wang et al. [5]), but none of the in-depth research was found openly about BTM used in high-speed lines.

This paper discussed the high-speed adaptability assessment issues through dynamic characteristics modelling and simulation. Section 2 described the dynamic characteristics modelling at four resolution layers, ranging from low to very high resolution. The relationship between BTM dynamic characteristics and the applicable train speed was verified by test data in Section 3. Finally, Section 4 formulated high speed adaptability evaluation criteria.

\section{BTM dynamic characteristics modelling}

\subsection{Train control system simulation requirement}

The train control system is a real-time distributed complex system. The simulation requirement differs as far as different end-users with a diversified focus are concerned. For example, operators own different viewpoints from suppliers, as do maintainers and constructors. The intention to adopt simulation varies from functional test to interoperability test, control strategy optimization, safety assessment, efficiency evaluation or training. Furthermore, the requirement also changes within different phases of system life cycle. Clearly, system modelling should satisfy such diversified needs. Classification of the simulation requirement should be considered deliberately; proven technology, i.e., the multi-resolution modelling method, was supposed to be a good choice.

\subsection{Multi-resolution BTM modelling}

\subsubsection{Overview}

In general, a train control simulation system is capable of reproducing the movement and operation status of trains despatched in pursuit over a pilot line with no less than three stations. In this context, three kinds of simulator are usually required, including the multi-train simulator, single train simulator and component Hardware-In-the-Loop (HIL) simulator. A multi-train simulator is able to simulate multiple trains running by their own working plan simultaneously. A single train simulator is often used to manifest the detailed behaviour of a train and its onboard equipment, especially the driver-machine interaction. The component HIL simulator is an effective test rig for the interoperability test, providing the HIL test environment for a specified component, such as BTM, Train Interface Unit, etc. Besides, component designers may additionally need the physical mechanism simulator to provide 
Table 1: $\quad$ Multi-resolution model of BTM dynamic characteristics.

\begin{tabular}{|c|c|c|c|c|}
\hline \multirow{2}{*}{ Target } & \multicolumn{2}{|c|}{ Dynamic characteristics } & Modelling & Resolution \\
\cline { 2 - 3 } & Layer & Feature & method & \\
\hline Multi-train simulator & Application & see 2.2 .2 & FFA & Low \\
\hline Single train simulator & Protocol & see 2.2 .3 & FFA & Medium \\
\hline BTM HIL simulator & Signal & see 2.2 .4 & TBM & High \\
\hline $\begin{array}{c}\text { Physical mechanism } \\
\text { Simulator }\end{array}$ & Filed & see 2.2 .5 & FEM & Very High \\
\hline
\end{tabular}

the finest granularity emulation of the electromagnetic coupling characteristics happening at the component interface. Table 1 shows the BTM dynamic characteristics model at these four resolution levels. A detailed explanation of Table 1 is as follows.

\subsubsection{Dynamic characteristics modelling at the application layer}

The BTM model at this layer has low resolution. The Functional Failure Analysis (FFA) (Nicholson [6]) method is used to analyse the features presented in the multi-train simulation process. We apply the five typical guide words ("Commission", "Omission", "Early", "Late" and "Value") to the failure analysis of the major top level function of BTM, i.e. the telegram reporting function. Getting rid of the meaningless outcome, the accurate description of five derived malfunctions are: the transmission of an erroneous telegram interpretable as correct, the loss of the telegram intended for full performance, erroneous reporting of a valid telegram in a different track, transmission of the valid telegram before the time window, transmission of the valid telegram after the time window. We believe, in addition to the normal function, the derived malfunctions depict properly the highest level BTM characteristics.

\subsubsection{Dynamic characteristics modelling at the protocol layer}

The communication protocol between the BTM and the Vital Computer (VC) onboard is the major concern of the single train simulator at this layer. We cannot make a general discussion of the dynamic communication behaviour, as the protocol is supplier dependent. However, FFA should be a suitable way to model it. For example, the guide word "Value" can be used to the communication baud rate and CRC result.

\subsubsection{Dynamic characteristics modelling at the signal layer}

It is necessary for the BTM HIL simulator to model the received up-link balise signal under specific train speed in the laboratory while the antenna and simulated balise are kept still. Test Based Modelling (TBM) is suggested at this layer, which is the same as the transmission test procedure described in Test Specification for Eurobalise Form Fit Function Interface Specification (FFFIS) SUBSET-085-v2.2.2 [7]. Firstly, the tele-power radiation pattern is evaluated by recording the flux of the tele-power signal at every geometrical test position. Secondly, the threshold curve is recorded while the BTM functions well with 
respect to the strength of the received up-link signal. An example of the radiation pattern and threshold curve is shown in Fig. 1. Then, the up-link signal for the dynamic test is created by a certain algorithm SUBSET-085-v2.2.2 [7], based on the results of two previous steps. The dynamic up-link signal can be generated by taking all the environmental factors into account, such as lateral displacement, debris, mounting height and speed. It is also important that the dynamic up-link signal can be used for BTM testing under standstill conditions. Actually, the signal is created by converting the up-link signal threshold at the diversified geometrical test position to the signal envelope at $(0,0$, maximum height). The BTM HIL simulator can be realized thus far (Zhen and Zhao [4] and Wang et al. [5]). Taking advantage of the BTM HIL simulator, we can experiment on the specific BTM. The following data can be acquired: (1) telegram and user data; (2) location report; (3) number of non-overlapping good telegrams; (4) BTM function reporting time.

These data can be further expressed graphically with respect to operational speed or any other factor of interest; then the corresponding BTM behaviour may be modelled, as in Section 3. In this process, the following two concepts were defined.

1) Static contact length: $L_{s}$. This denotes the effective action length along with the $\mathrm{X}$-axis direction, while the BTM can correctly decode and output the balise telegram within the main lobe region under the specified static conditions. For the weakest balise, the formula is:

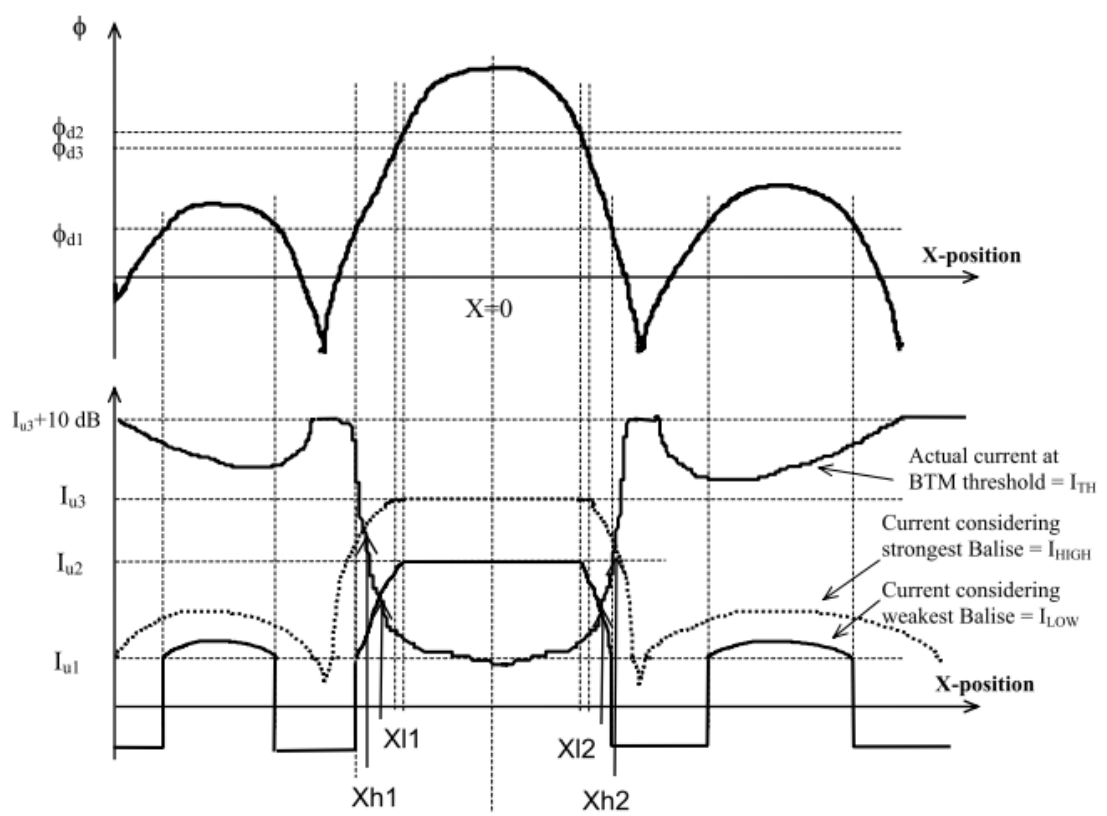

Figure 1: Example of signal radiation pattern and threshold curve. 


$$
L_{\mathrm{s}}=X_{l 2}-X_{l 1}, \quad\left(X_{l 1}, X_{l 2} \in\left[x: I_{t h}(x)-I_{l o w}(x)=0\right]\right)
$$

where $X_{l l}, L_{l 2}, I_{\text {th }}$ and $I_{\text {low }}$, are as shown in Fig. 1 .

2) Dynamic contact length: $L_{d}$. This denotes the effective action length along with the X-axis direction, while the BTM can correctly decode and output the balise telegram within the main lobe region under the specified dynamic conditions. For the weakest balise, the formula is:

$$
\begin{gathered}
L_{d}=X_{\mathrm{L} 2}-X_{L 1}-\left(T_{b t m}+T_{d i s}+T_{d e b}+T_{\text {end }}\right) \cdot v, \quad\left(X_{L 1}, X_{L 2} \in\left[x: I_{t h}(x)-I_{B d l}(x)=0\right]\right) \\
L_{d}=L_{s}-\left(T_{b a l}+T_{b t m}+T_{d i s}+T_{d e b}+T_{e m d}\right) \cdot v
\end{gathered}
$$

where $T_{b a l}$ denotes the start-up time of the balise; $T_{b t m}$ denotes the start-up time of the BTM decoding function together with the Antenna Unit; $T_{d i s}$ denotes the delay time due to random displacement between the antenna and balise; $T_{d e b}$ denotes the delay time due to change of debris; $T_{\text {emd }}$ denotes the delay time due to changes of the spatial electromagnetic environment.

\subsubsection{Dynamic characteristics modelling at the field layer}

The Finite Element Method (FEM) is suitable for modelling the dynamic behaviour of the BTM in the highest resolution. BTM designers need to know more about the electromagnetic field distribution and quantitative coupling property where specific application scope, such as train speed and balise discreteness, is concerned.

\section{Speed factor in BTM dynamic characteristics}

A high resolution model built at the signal layer provides a suitable basis for the discussion of the influence of operational speed upon BTM dynamic characteristics. The BTM HIL simulator described in Section 2.2.4 is capable of acquiring a "number of non-overlapping good telegrams (denoted as $N_{g}$ )" with respect to "simulated train speed". Taking the BTM developed by Beijing Jiaoda Signal Technology Co., Ltd (BJST) as an example, test data were as shown in Figs. 2 and 3. The nominal mounting height for the antenna unit is $463 \mathrm{~mm}$, i.e. $\mathrm{H}=463 \mathrm{~mm}$. The lateral displacement is $110 \mathrm{~mm}$ in all test cases, i.e. $\mathrm{Y}=110 \mathrm{~mm}$. Every test case was repeated 10 times. The test result of each repeat had an effect on the final test results. The test result was summarized as follows.

- The BTM received a fewer number of telegrams as speed was increased.

- Ice on the antenna had no significant effect on the BTM function (Fig. 2).

- Compared with the condition of the metallic plane under the simulated balise, the BTM received more than 0.5 frames of telegram at nominal condition for the weakest balise.

- Compared with the weakest balise, the strongest balise contributed 1 to 2 more frames of telegram for the BTM function (Fig. 3).

The BTM dynamic characteristics had significant relation with train speed while the number of non-overlapping good telegrams was evaluated. To be clearer, the dynamic contact length was evaluated instead, as shown in Fig. 4 for debris test cases. Obviously, the dynamic contact length decreased $26 \mathrm{~mm}$ per $100 \mathrm{Km} / \mathrm{h}$ averagely when speed was increased. Let $L_{d}$ denote the dynamic 
contact length in metres, whereas $L_{s}$ is the static contact length, namely the contact length when the speed was approaching zero. Let $v$ denote the speed in $\mathrm{m} / \mathrm{s}$ and $\tau$ denote the speed factor. The following formula was derived by linear curve fitting,

$$
L_{d}=L_{s}-\tau \cdot v
$$

where $\tau=9.36 \times 10^{-4} s$. Clearly, this formula is the same as the formula (3). The speed factor $\tau$ is actually the effect of the start-up time of the balise and the BTM function together with the Antenna Unit, as well as the disturbance factor of $T_{d i s}, T_{d e b}$ and $T_{e m d}$ defined in formula (3). From another point of view, $L_{d}$ is a product of the dynamic action time and speed, where the dynamic action time can be derived from $N_{g}$, as shown in formula (5). Here, 1023 is the number of bits in one frame of the balise telegram; and 564480 is the mean data rate in bits per second.

$$
L_{d}=\frac{N_{g} \cdot 1023 \cdot v}{564480}
$$

Combining formulas (4) and (5), we can get the relation between $N_{g}$ and $v$, which is the mathematical expression of Figs. 2 and 3.

$$
N_{g}=\frac{564480}{1023}\left(\frac{L_{s}}{v}-\tau\right)
$$

\section{High speed adaptability evaluation criteria}

High speed adaptability for the BTM can be evaluated based on the above modelling and reasoning results. Firstly, the number of non-overlapping good telegrams for the specified BTM and antenna under specific conditions, namely $N_{g}$, is an important figure. It is in inverse proportion to speed, as shown in formula (6). Secondly, according to mathematics statistics theory, the BTM decoding failure probability, denoted as $P_{b e r}$, is able to be derived when the nonoverlapping telegrams with a total number of $N_{t}$ is received under the contaminated environment with certain Bit Error Rates (BERs) [2]. Supposing

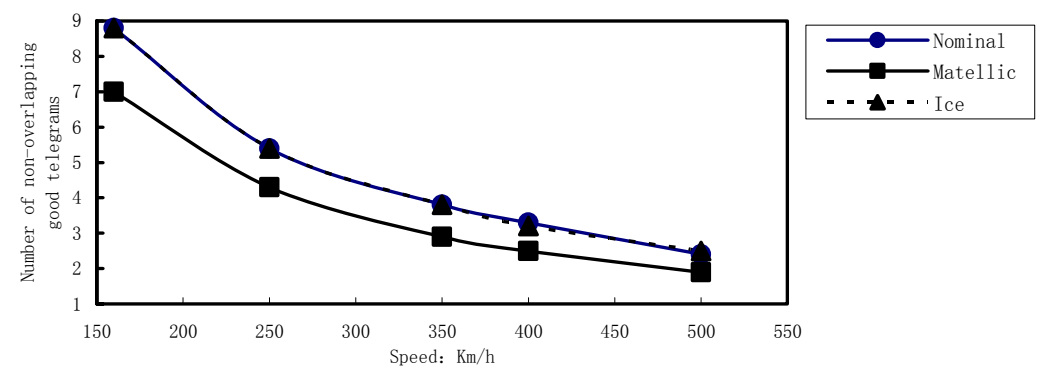

Figure 2: $\quad$ BTM performance under debris $(\mathrm{H}=463 \mathrm{~mm} / \mathrm{Y}=110 \mathrm{~mm})$. 


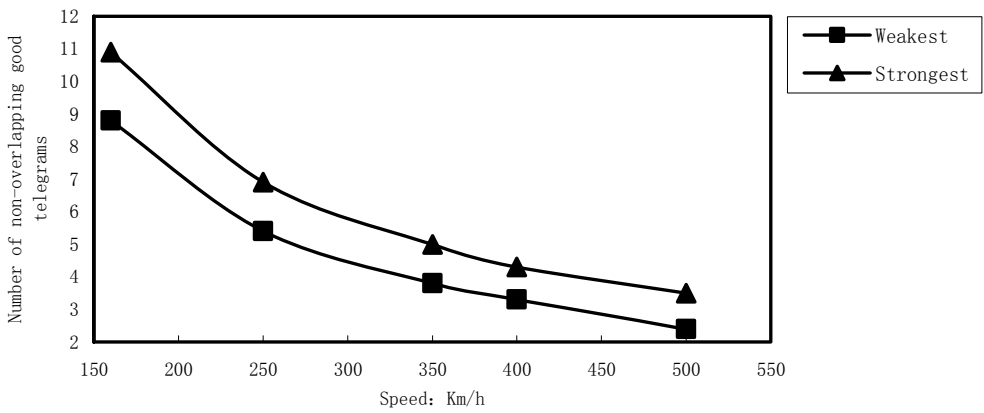

Figure 3: $\quad$ BTM performance with respect to the strongest and weakest balise $(\mathrm{H}=463 \mathrm{~mm} / \mathrm{Y}=110 \mathrm{~mm})$.

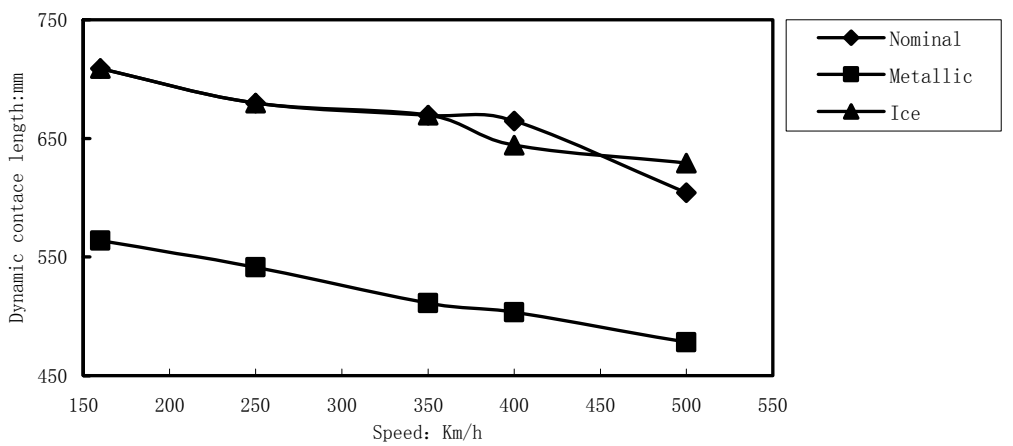

Figure 4: Dynamic contact length under debris $(\mathrm{H}=463 \mathrm{~mm} / \mathrm{Y}=110 \mathrm{~mm})$.

Table 2: $\quad$ Decoding failure probability with respect to $N_{t}$.

\begin{tabular}{|c|c|c|c|}
\hline$P_{\text {ber }}$ & $1 \mathrm{E}-6$ & $2.71 \mathrm{E}-8$ & $9.20 \mathrm{E}-11$ \\
\hline$N_{t}$ & 2.0 & 3.0 & 3.5 \\
\hline
\end{tabular}

that the BER within the dynamic contact length is evenly 10E-6, the relationship between the two figures is expressed in Table 2 (Zhao et al. [8]). A basic receiver is assumed to be used for the BTM in the above cases.

Thirdly, $P_{b e r}$ can be determined by specific availability targets of certain lines within the entire specified range of railway conditions and train speeds. For example, if the mean BTM failure rate due to BER will be less than 2.66 times per annum, a mean figure of 10E8 balise passages with error free telegrams delivered by the BTM to the VC should be ensured in the Beijing-Shanghai High Speed Line (the line is supposed to be about $1400 \mathrm{Km}, 2$ balises per kilometre, 260 trains per day. The annual number of balise passages will be 2.66E8). Then, $P_{b e r}$ should be $10 \mathrm{E}-8$. Fourthly, we can find the required total number of nonoverlapping telegrams $N_{t}$, approximately 3.0 in this instance. Finally, if we determined $N_{g}$ by the BTM HIL simulator in the laboratory, where BER can be controlled to zero, $N_{g}$ should be no less than $N_{t}$ in principle. Therefore, the high 
speed adaptability evaluation criteria for the BTM are simplified to the comparison of $N_{g}$ and $N_{t}$.

\section{Conclusion}

BTM dynamic characteristics can be modelled at four resolution layers by FFA, FFA, TBM and FEM, respectively. For specified BTMs and antennas under specific conditions, the number of non-overlapping good telegrams is in inverse proportion to speed, whereas the dynamic contact length decreases linearly with speed. High speed adaptability for the BTM can be evaluated by comparison of the number of non-overlapping good telegrams received by the BTM with the predetermined value. This value is derived from specific availability targets of certain lines.

\section{Acknowledgements}

We gratefully acknowledge the financial support provided by The National Natural Science Foundation of China (Grant No. 60736047) and the National 863 High-tech R\&D Program (Grant No. 0912JJ0104). We also would like to thank the CEDEX Eurobalise Laboratory Spain for providing the test facilities and test reports for the contractual BTM test activities.

\section{References}

[1] Ministry of Railway China, KeJiYun [2008] No.143, Balise Application Principle for CTCS Level 2 (V1.0). Train Control System Specification for Passenger Dedicated Line, Ministry of Railway, Beijing, China, 2008.

[2] Union Industry of Signaling, SUBSET-036-V2.4.1 Form Fit Function Interface Specification for Eurobalise. Brussels: Alstom Ansaldo Bombardier Invensys Siemens Thales, 2007.

[3] Yang, Z., Fan, P., \& Xue, R., Balise System Used in High Speed or Speedincrease Line. China Railway Science, Beijing, 23(2), pp.42-47, 2002

[4] Zhen, J., \& Zhao, H., Research on Balise Transmission Module Test System, Journal of Beijing Jiaotong University, 32(2), pp. 80-83, 2008.

[5] Wang, R., Zhao, H., \& Wang, S., Research on Up-link Signal Simulator Used for BTM Test in Balise system. Journal of the China Railway Society, Beijing, 30(6), pp. 46-50, 2008.

[6] Nicholson, M., Lecture Note: Fundamental Safety Engineering, University of York, 2008.

[7] Union Industry of Signalling, SUBSET-085-V2.2.2 Test Specification for Eurobalise Form Fit Function Interface Specification. Alcatel Alstom Ansaldo signal Bombadier Invensys Rail Siemens: Brussels, 2007.

[8] Zhao, H., Li, W., Zhao, M., \& Liu, Z., Dynamic Characteristic and HighSpeed Adaptability of Balise Onboard Equipment. (To be published) China Railway Science, Beijing, China, 2010. 